\title{
CONSISTENCY OF THE CONDITIONS DETERMINING KOLLEKTIVS*
}

\author{
BY
}

\author{
ARTHUR H. COPELAND $\dagger$
}

1. Fundamental concepts. With the establishment of the consistency of the conditions determining collectives, $\ddagger$ we obtain a sound basis for the theory of probability. We shall see that certain slight modifications of these conditions will be necessary in order to establish consistency but these modifications will destroy none of the intrinsic value of von Mises' theory. In those alternative systems for which consistency has been established, the conditions are so much weaker than those of von Mises that much of the value of his theory has been lost.

The collective is a mathematical idealization of a sequence of occurrences (measurements, tosses of a coin, etc.). A collective $\mathrm{K}$ consists of an infinite sequence of elements (points of some space $S$ ). Thus $\S$

$$
K=e^{(1)}, e^{(2)}, e^{(3)}, \cdots
$$

The points $m$ of $S$ are called labels (Merkmale) and the space $S$ the label space (Merkmalraum).

2. Fields and distribution functions. Let $F$ be a set such that its elements are subsets of the space $S$ and such that the sum, product, and difference (when defined) of any two of its elements also belong to $F$. Also let the space $S$ be an element of $F$. Then $F$ is called a field. $\uparrow$ Associated with every element $A$ of $F$, is a probability $\pi(A)$ satisfying the following conditions.

1. If $A$ and $B$ are any two mutually exclusive elements of $F$ (i.e., $A \cdot B$ is the null set 0$)$, then $\pi(A+B)=\pi(A)+\pi(B)$.

2. For every element $A$ of $F, 0 \leqq \pi(A)$.

* Presented to the Society, June 22, 1933; received by the editors September 25, 1935.

$\dagger$ Guggenheim Fellow.

$\ddagger$ The concept of collective (Kollektiv) is due to von Mises. See R. von Mises I, II, III. References to the literature are given at the end of this paper.

$\S$ At a later point we shall state the restrictions which must be imposed upon $K$ in order that it may be a collective.

$\|$ The letters $m$ and $e$ are used to refer respectively to points of $S$ and elements of $K$. Two $e$ 's with different superscripts may be equal to the same element $m$ of $S$. For example, in the tossing of a coin, the space $S$ consists of two elements $m_{1}$ and $m_{2}$ (heads and tails) and in general each of these elements is repeated an infinite number of times in the corresponding collective.

ๆ Kolmogoroff I. 
3. $\pi(S)=1$.

4. If $A_{1}, A_{2}, A_{3}, \cdots$ is any sequence of elements of $S$ such that $A_{1}>A_{2}$ $>A_{3}>\cdots$ and $\prod_{n=1}^{\infty} A_{n}=0$, then $\lim _{n \rightarrow \infty} \pi\left(A_{n}\right)=0$.

The function $\pi(A)$ satisfying the above conditions is a generalized measure function, but in this paper it will be referred to as a distribution function (Verteilung).

3. Product spaces and independence. Let $S_{1}, S_{2}, \cdots, S_{\alpha}$ be $\alpha$ label spaces. We shall denote the product space $S$ by $S_{1} \times S_{2} \times \cdots \times S_{\alpha}$. If $m_{1}, m_{2}, \cdots, m_{\alpha}$ are respectively points of the spaces $S_{1}, S_{2}, \cdots, S_{\alpha}$, then $m=m_{1} \times m_{2} \times m_{3} \times \cdots \times m_{\alpha}$ is an element of the product space $S$. The element $m$ may be considered as a point whose coordinates are $m_{1}, m_{2}, \cdots, m_{\alpha}$.

Let $K_{i}$ be a collective whose label space is $S_{i}(i=1,2, \cdots, \alpha)$ and let $e_{i}^{\left({ }^{(n)}\right.}$ be the $n$th term of the collective $K_{i}$. We shall form a collective $K$ whose $n$th element $e^{(n)}$ is given by the equation $e^{(n)}=e_{1}^{(n)} \times e_{2}^{(n)} \times e_{3}^{(n)} \times \cdots \times e_{\alpha}^{(n)}$. We shall call $K$ the product collective and shall denote it by the expression $K_{1} \times K_{2}$ $\times K_{3} \times \cdots \times K_{\alpha}{ }^{*}$ von Mises designates as a Verbindung the operation by which the product collective is formed.

Let $F_{i}$ be a field of the space $S_{i}$ and let $A_{i}$ be a set of the field $F_{i}$ $(i=1,2,3, \cdots, \alpha)$. Let $A$ be the product set $A_{1} \times A_{2} \times \cdots \times A_{\alpha}$. Then $A$ shall contain all points $m=m_{1} \times m_{2} \times \cdots \times m_{\alpha}$ such that $m_{i}$ belongs to $A_{i}$. The product field $F=F_{1} \times F_{2} \times \cdots \times F_{\alpha}$ shall contain all product sets which can be formed from the fields $F_{1}, F_{2}, \cdots, F_{\alpha}$. It shall also contain sums, logical products, and differences of such sets. Thus a product field is itself a field.

Let us suppose that the collectives $K_{1}, K_{2}, \cdots, K_{\alpha}$ possess distribution functions $\pi_{1}\left(A_{1}\right), \pi_{2}\left(A_{2}\right), \cdots, \pi_{\alpha}\left(A_{\alpha}\right)$ and that the product collective $K=K_{1} \times K_{2} \times \cdots \times K_{\alpha}$ possesses the distribution function $\pi(A)$. Then the collectives $K_{1}, K_{2}, \cdots, K_{\alpha}$ will be called independent provided

$$
\pi(A)=\pi\left(A_{1} \times A_{2} \times \cdots \times A_{\alpha}\right)=\pi_{1}\left(A_{1}\right) \cdot \pi_{2}\left(A_{2}\right) \cdots \pi_{\alpha}\left(A_{\alpha}\right) .
$$

It will be noted that the order of multiplication is immaterial to the definition of independence. Moreover if the collectives $K_{1}, K_{2}, \cdots, K_{\alpha}$ are independent and if $K_{n_{1}}, K_{n_{2}}, \cdots, K_{n_{\alpha}}$ is any subset of these collectives, then the collectives of this subset are independent. In order to define the independence of an infinite set of collectives, we shall place a restriction upon the product field. Namely if $A_{1} \times A_{2} \times \cdots \times A_{\alpha} \times \cdots$ is any product set of the product field, then only a finite number of the sets $A_{i}$ will be proper subsets of the corresponding spaces $S_{i}$. The remaining sets $A_{i}$ will be equal to the corresponding spaces $S_{i}$. Thus an infinite set of collectives is an independent set if and only if every finite subset is an independent set.

* Dörge I. 
4. Expected value and functions of collectives. The expected value $p(K)$ of a collective $K$ is defined as follows. Let

$$
p_{n}(K)=\sum_{i=1}^{n} e^{(i)} / n *^{*}
$$

Then

$$
p(K)=\lim _{n \rightarrow \infty} p_{n}(K) .^{*}
$$

Functions of collectives are defined in the following manner. Let $f(m)$ be any function defined for all points of $S$ and let $f(K)$ be the sequence

$$
f(K)=f\left(e^{(1)}\right), f\left(e^{(2)}\right), f\left(e^{(3)}\right), \cdots .
$$

This transforms the collective $K$ into a new collective $f(K)$ and is a generalization of the operation von Mises calls Mischung. $\dagger$ Functions of any number of collectives are automatically defined in terms of the concepts of product spaces and product collectives.

A function which is of particular importance in this theory is the fundamental function of point sets. $\ddagger$ Let $A$ be any element of $F$ and let $\phi_{A}(m)$ be the fundamental function of the set $A$. Then $\phi_{A}(m)$ has the value 1 when $m$ is a point of $A$ and the value 0 otherwise. In the case of the product space $S=S_{1} \times S_{2} \times \cdots \times S_{\alpha}$, the fundamental function satisfies the equation

$$
\phi_{A_{1} \times A_{2} \times \cdots \times A_{\alpha}}\left(m_{1} \times m_{2} \times \cdots \times m_{\alpha}\right)=\phi_{A_{1}}\left(m_{1}\right) \cdot \phi_{A_{2}}\left(m_{2}\right) \cdots \phi_{A \alpha}\left(m_{\alpha}\right) .
$$

The expected value $p\left[\phi_{A}(K)\right]$ of the collective $\phi_{A}(K)$ is the probability with respect to the collective $K$, of obtaining a point of the set $A$. That is

$$
p\left[\phi_{A}(K)\right]=\pi(A) .
$$

This may be seen as follows. The summation $\sum_{i=1}^{n} \phi_{A}\left(e^{(i)}\right)$ is the number of times that elements of $A$ occur in the first $n$ terms of $K$. Hence $p_{n}\left[\phi_{A}(K)\right]$ is the success ratio for elements of $A$ in the first $n$ terms of $K$. The limit of this success ratio $p\left[\phi_{A}(K)\right]$ is the probability. The collective is said to possess a distribution with respect to a field $F$ provided the limit $\pi(A)$ exists for all sets $A$ of $F$ and provided $\pi(A)$ satisfies condition 4 of $\$ 2$. It will be observed that conditions 1,2 , and 3 are satisfied automatically.

5. Selections. We shall now define an operation called selection (Aus-

* The expressions $p_{n}(K)$ and $p(K)$ will be used only when the corresponding space $S$ is such that addition and division by a number have a meaning, and $p(K)$ will be used only when the limit exists. $\dagger$ von Mises reserves the term Mischung for the case where the operation is single-valued and its inverse is multi-valued.

$\ddagger$ de la Vallée Poussin I. 
wahl). Let $n_{1}, n_{2}, n_{3}, \cdots$ be an increasing sequence of positive integers. From any collective $K$ we can form a new collective $K^{\prime}$ by selecting the $n_{1}$ st, $n_{2}$ nd, $\cdots$ terms of $K$. Thus

$$
K^{\prime}=e^{\left(n_{1}\right)}, e^{\left(n_{2}\right)}, e^{\left(n_{3}\right)}, \cdots \text {. }
$$

It is convenient to introduce a notation for this operation. Let $x=x^{(1)}, x^{(2)}$, $x^{(3)}, \cdots$ be a sequence whose terms $x^{\left(n_{1}\right)}, x^{\left(n_{2}\right)}, \cdots$ are all 1 's and the rest of whose terms are all 0 's. Thus $x$ is a sequence similar to $K$ and the associated label space consists of the elements 1 and 0 . We shall denote the operation of selection by the equation $K^{\prime}=K \subset x$. $^{*}$ It should be observed that $f(K \subset x)=f(K) \subset x$.

A selection $x$ may be regarded as a number written in the binary scale, with digits $x^{(1)}, x^{(2)}, \cdots$ and with the radix point placed immediately before the first digit. Any number in the interval from 0 to 1 ( 0 excluded) can represent a selection. In the case of the ambiguous representation, the ambiguity is resolved by means of the condition that an infinite number of the terms of the collective must be selected and hence an infinite number of the digits of $x$ must be equal to 1 .

Let us operate on the collective $K$ by a selection $x_{1}$, obtaining a new collective $K^{\prime}=K \subset x_{1}$. Let us operate on the resulting collective $K^{\prime}$ by a selection $x_{2}$, obtaining $K^{\prime \prime}=K^{\prime} \subset x_{2}$. Let us suppose that $x_{1}$ selects the $n_{1}$ st, $n_{2}$ nd, $\cdots$ terms of $K$ and that $x_{2}$ selects the $\nu_{1}$ st, $\nu_{2}$ nd, . . terms of $K^{\prime}$. The resultant of the two selections is equivalent to a single operation $x$ which selects the $n_{\nu_{1}} \mathrm{st}, n_{\nu_{2}} \mathrm{nd}, \cdots$ terms of $K$. That is $\left(K \subset x_{1}\right) \subset x_{2}=K \subset x$.

A set $D$ of selections will be called a fundamental set provided the resultant of any two selections of $D$ is a selection of $D$ and provided $D$ contains the identity selection $1=1,1,1, \cdots$. A fundamental set fails to be a group since a selection can have no unique inverse. The set consisting only of the identity is an example of a fundamental set. A second example is the set consisting of all possible selections. As a third example we shall construct a fundamental set $D_{0}$ which will be used to define independence of terms of a collective. We shall denote the elements of $D_{0}$ by the symbol $x_{r, n}$ where $r$ and $n$ are positive integers. The operator $x_{r, n}$ selects the $r$ th, the $(r+n)$ th, the $(r+2 n)$ th, the $(r+3 n)$ th, $\cdots$ terms of a collective $K$. The resultant of the operation $x_{r, n}$ followed by the operation $x_{\rho, \nu}$ is equivalent to the operation $x_{R, N}$ where $R=r+(\rho-1) n$ and $N=n \nu$. The selection $x_{1,1}$ is the identity.

6. Independence of the terms of a collective. If a well balanced coin is thrown twice, the probability of obtaining heads on the first throw and tails

* The symbol $\subset$ is the inverted implication sign and is interpreted by the word "if." For this interpretation, see Copeland I. 
on the second is $1 / 4$. To verify this experimentally, it would be natural to throw a coin twice and observe whether or not heads were obtained on the first trial and tails on the second. Then repeat the experiment a number of times. When $n$ such experiments have been made, the coin has been thrown $2 n$ times. That is, there are determined $n$ groups of two throws each. These throws constitute the first $2 n$ terms of a collective $K$. Let $m_{1}$ and $m_{2}$ represent respectively the labels heads and tails. Then $\phi_{m_{1}}\left(K \subset x_{1,2}\right)$ is a sequence of 1's and 0 's such that a 1 occurs when heads is obtained on the first throw of a group of two, and a 0 otherwise. Similarly $\phi_{m_{2}}\left(K \subset x_{2,2}\right)$ is a sequence representing tails on the second throw of each group. The product $\phi_{m_{1}}\left(K \subset x_{1,2}\right)$ $\cdot \phi_{m_{2}}\left(K \subset x_{2,2}\right)$ is the sequence whose probability we are investigating. We should expect the success ratio $p_{n}\left[\phi_{m_{1}}\left(K \subset x_{1,2}\right) \cdot \phi_{m_{2}}\left(K \subset x_{2,2}\right)\right]$ to be approximately $1 / 4$ and the probability $p\left[\phi_{m_{1}}\left(K \subset x_{1,2}\right) \cdot \phi_{m_{2}}\left(K \subset x_{2,2}\right)\right]$ to be equal to $1 / 4$.

In a like manner we should have

$$
\begin{aligned}
p\left[\phi_{m_{2}}\left(K \subset x_{1,3}\right) \cdot \phi_{m_{1}}\left(K \subset x_{2,3}\right) \cdot \phi_{m_{1}}\left(K \subset x_{3,3}\right)\right] & \\
& =p\left[\phi_{m_{2}}\left(K \subset x_{1,3}\right)\right] \cdot p\left[\phi_{m_{1}}\left(K \subset x_{2,3}\right)\right] \cdot p\left[\phi_{m_{1}}\left(K \subset x_{3,3}\right)\right]=1 / 8,
\end{aligned}
$$

since the probability of tails on the first throw, heads on the second, and heads on the third is $1 / 8$. The same probability should be obtained for the combinations $m_{1}, m_{1}, m_{1} ; m_{1}, m_{1}, m_{2} ; m_{1}, m_{2}, m_{1} ; \cdots ; m_{2}, m_{2}, m_{2}$. That is, the sequences $K \subset x_{1,3}, K \subset x_{2,3}, K \subset x_{3,3}$ should be independent. We shall say that the terms of a collective $K$ are independent provided the sequences $K \subset x_{1, n}, K \subset x_{2, n}$, $\cdots, K \subset x_{n, n}$ are independent for every positive integer $n$.

7. Contractions. If $K$ is any collective and $A$ is any subset of the corresponding label space, then $\phi_{A}(K)$ is a sequence of 1's and 0's and hence can be used as a selection operator. The operation $K \subset \phi_{A}(K)$ is called a contraction (Teilung).* By means of the contraction, the collective $K$ is transformed into a new collective with the contracted label space $A$.

The operations of selection and contraction can be combined. Thus we may perform a contraction $K \subset \phi_{A}(K)$ on the collective $K$ and then operate on the resulting collective by a selection $x$. The resultant of the two operations is denoted as follows, $K \subset \phi_{A}(K) \cdot c \cdot x$. The two dots are used as brackets. $\dagger$ Everything to the left of the first dot is understood to be enclosed in one bracket and the $x$ to the right of the second dot is enclosed in another. Thus the expression $K \subset \phi_{A}(K) \cdot \subset \cdot x$ means $\left[K \subset \phi_{A}(K)\right] \subset[x]$. That is, the dots indicate that the contraction is performed before the selection. In case

* Contraction is not intended as a translation of the word Teilung but it is descriptive of the operation.

$\dagger$ Lewis and Langford I. 
further bracketing is required, two dots may be used to indicate a stronger bracket than one dot. For example, $K \subset x_{1} \cdot c \cdot \phi_{A}\left(K \subset x_{1}\right): \subset: x_{2}$ means $\left\{\left[K \subset x_{1}\right] \subset\left[\phi_{A}\left(K \subset x_{1}\right)\right]\right\} \subset\left\{x_{2}\right\}$. It should be observed that $K \subset \phi_{A}(K)$ . $\subset \phi_{B}\left[K \subset \phi_{A}(K)\right]=K \subset \phi_{A \cdot B}(K)$ for any two sets $A$ and $B$. That is, the resultant of two contractions is a contraction.

8. Restrictions on collectives. von Mises places the following restrictions on the collective.

(M1) The limit $p\left[\phi_{A}(K)\right]$ exists for every element $A$ of the field $F$.

(M2) If $A$ and $B$ are any two mutually exclusive elements of $F$ such that $p\left[\phi_{A}(K)\right]$ and $p\left[\phi_{B}(K)\right]$ are not both 0 and if $x$ is any selection of the fundamental set $D$, then the limits $p\left\{\phi_{A}\left[K \subset \phi_{A+B}(K) \cdot \subset \cdot x\right]\right\}$ and $p\left\{\phi_{B}\left[K \subset \phi_{A+B}(K) \cdot \subset \cdot x\right]\right\}$ exist and

$p\left\{\phi_{A}\left[K \subset \phi_{A+B}(K) \cdot \subset \cdot x\right]\right\} / p\left\{\phi_{B}\left[K \subset \phi_{A+B}(K) \cdot \subset \cdot x\right]\right\}=p\left[\phi_{A}(K)\right] / p\left[\phi_{B}(K)\right]$.

(M3) The set $D$ contains all selections which are independent of $K$.

(M4) The field $F$ contains all possible subsets of the label space $F$.

The first restriction states that the collective must possess a distribution. The second restriction can be most easily understood by specializing the operators. Let us consider the case in which $x$ is the identity selection. The terms of $K \subset \phi_{A+B}(K)$ belong either to $A$ or to $B$ and hence

$$
p\left\{\phi_{A}\left[K \subset \phi_{A+B}(K)\right]\right\}+p\left\{\phi_{B}\left[K \subset \phi_{A+B}(K)\right]\right\}=1 .
$$

From this equation and from condition 2 , it follows by simple algebra that

$$
p\left\{\phi_{A}\left[K \subset \phi_{A+B}(K)\right]\right\}=p\left[\phi_{A}(K)\right] /\left\{p\left[\phi_{A}(K)\right]+p\left[\phi_{B}(K)\right]\right\}
$$

and

$$
p\left\{\phi_{B}\left[K \subset \phi_{A+B}(K)\right]\right\}=p\left[\phi_{B}(K)\right] /\left\{p\left[\phi_{A}(K)\right]+p\left[\phi_{B}(K)\right]\right\} .
$$

That is, the probability that a term of $K$ will lie in $A$ if it lies in $A$ or $B$ is equal to the probability that a term of $K$ will lie in $A$ divided by the probability that it will lie in either $A$ or $B$. Next let us specialize by letting $A+B=S$. In this case the contraction reduces to the identity. Hence it is easily seen that condition (M2) implies that the distribution of a collective is invariant under the operation of all selections of the set $D$.

There has been considerable discussion concerning the meaning of condition (M3).* In fact, this seems to be the most serious difficulty in von Mises' theory. Since the objections have been adequately treated in other papers it is not necessary to repeat them here. We shall however give an alternative restriction to which these objections do not apply.

* Kamke I. 
It is also necessary to weaken condition (M4). I have proved that conditions (M1) and (M4) cannot both be satisfied if the distribution function is absolutely additive and absolutely continuous. ${ }^{*}$ Thus, for example, these conditions exclude any collective whose probability is distributed in accordance with the normal law.

9. An alternative set of conditions. We shall denote by $C[D, \pi(E)]$ the set of all collectives associated with a given fundamental set $D$ and a distribution function $\pi(E)$ defined on a field $F$. The range of $\pi(E)$ determines the field. We shall demand that the set of collectives belonging to $C[D, \pi(E)]$ satisfy the following conditions.

(C1) $p\left[\phi_{E_{1}}\left(K \subset x_{r_{1}, n}\right) \cdot \phi_{E_{2}}\left(K \subset x_{r_{2}, n}\right) \cdots \phi_{E_{h}}\left(K \subset x_{r_{h}, n}\right)\right]=\pi\left(E_{1}\right) \cdot \pi\left(E_{2}\right)$ $\cdot \pi\left(E_{3}\right) \cdots \pi\left(E_{h}\right)$ for every set of integers $r_{1}, r_{2}, r_{3}, \cdots, r_{h}, n$ such that $0<r_{1}<r_{2}<\cdots<r_{h} \leqq n$ and for every set $E_{1}, E_{2}, E_{3}, \cdots, E_{h}$ of elements of $F$.

(C2) $K \subset \phi_{A}(K)$ is an element of $C[D, \pi(E) / \pi(A)]$ for every element $A$ of $F$ such that $0<\pi(A)$.

(C3) $K \subset x$ is an element of $C[D, \pi(E)]$ for every element $x$ belonging to $D$.

The collectives satisfying these conditions also satisfy (M1) and (M2). Thus suppose we specialize condition (C1) by letting $h=r_{1}=n=1$. Then since $x_{1,1}$ is the identity selection, (C1) reduces to the condition $p\left[\phi_{E}(K)\right]$ $=\pi(E)$. Next let us observe that if $E_{1}$ and $E_{2}$ are any two mutually exclusive elements of $F$ such that $\pi\left(E_{1}\right)$ and $\pi\left(E_{2}\right)$ are not both zero, then conditions (C1), (C2), and (C3) imply that

$$
p\left\{\phi_{E_{1}}\left[K \subset \phi_{E_{1}+E_{2}}(K) \cdot \subset \cdot x\right]\right\}=\pi\left(E_{1}\right) / \pi\left(E_{1}+E_{2}\right)
$$

and

$$
p\left\{\phi_{E_{2}}\left[K \subset \phi_{E_{1}+E_{2}}(K) \cdot \subset \cdot x\right]\right\}=\pi\left(E_{2}\right) / \pi\left(E_{1}+E_{2}\right) .
$$

Conditions (C1), (C2), and (C3) are much stronger than (M1) and (M2). In the first place, (C1) demands that the terms of a collective be independent. Secondly, (C2) and (C3) enable us to operate on a collective by any finite group of selections and contractions, whereas condition (M2) only permits operation first with a contraction, then with a selection. Due to the recursive nature of (C2) and (C3), it is in general true that when one collective of the $C[D, \pi(E)]$ has been obtained, a continuum of such collectives is automatically determined.

It will be recalled that conditions (M3) and (M4) relate to the choice of the fundamental set $D$ and the field $F$. Whether or not there exist collectives

\footnotetext{
* Copeland II.
} 
belonging to $C[D, \pi(E)]$ depends upon the choice of $D$ and $F$. Thus, for example, if $D$ contains all possible selections and if $F$ contains two mutually exclusive elements $A$ and $B$ such that neither $\pi(A)$ nor $\pi(B)$ is equal to zero, then $C[D, \pi(E)]$ is null. In this paper we shall assume that $D$ is denumerable. The restriction which we shall place on $F$ will depend upon the type of label space. Thus if $S$ is finite, $F$ may be unrestricted. If $S$ is euclidean, we shall assume that $F$ consists of all subsets of $S$ whose frontier points are of Lebesgue measure zero.

10. Special fields. We shall consider certain special spaces and fields for which the corresponding collectives can be more easily constructed. The collectives thus obtained constitute a basic set in terms of which more general collectives can be formed. We shall construct a space $S$ consisting of points whose coordinates $y_{1}, y_{2}, \cdots, y_{\alpha}$ satisfy the inequalities $0 \leqq y_{i}<1$, $i=1,2, \cdots, \alpha$. We shall define a field $F$ for $S$. A set $A$ will belong to $F$ if and only if the frontier points of $A$ constitute a set of Lebesgue measure zero. It can readily be verified that $F$ is a field. We shall let $\pi(A)$ be any absolutely continuous distribution function whose range is $F$.

Since the collective is a sequence of points and hence denumerable, it is not necessary to use all of the space $S$. Hence we shall define a space $S^{\prime}$ consisting of a denumerable everywhere dense set of points in the space $S$. A subset $A^{\prime}$ of $S^{\prime}$ will be an element of the corresponding field $F^{\prime}$ if and only if there exists an element $A$ of $F$ such that $A \cdot S^{\prime}=A^{\prime}$. A distribution function $\pi^{\prime}\left(A^{\prime}\right)$ whose range is $F^{\prime}$ is defined by the equation $\pi^{\prime}\left(A \cdot S^{\prime}\right)=\pi(A)$.

It is necessary to show that the above distribution function is uniquely defined. In order to do this, we shall first prove that if $A^{\prime}$ is any element of $F^{\prime}$ and $A_{1}$ and $A_{2}$ are elements of $F$ such that $A_{1} \cdot S^{\prime}=A_{2} \cdot S^{\prime}=A^{\prime}$, then $A_{1}$ and $A_{2}$ differ by at most a set of points of measure zero. Let $f_{1}$ and $f_{2}$ be respectively the frontier points of $A_{1}$ and $A_{2}$ and let $f=f_{1}+f_{2}$. Then the sets $B_{1}=A_{1}-A_{1} \cdot f$ and $B_{2}=A_{2}-A_{2} \cdot f$ are open. Let $p$ be any point of the set $B_{1}$. Then it is possible to enclose $p$ in a spherical region $\sigma$ such that all points of $\sigma$ belong to $A_{1}$. Hence $\sigma$ must contain points of the set $A^{\prime}$. But all points of $A^{\prime}$ are also points of $A_{2}$. Hence $p$ is a limit point of points of $A_{2}$. Moreover $p$ is not a point of $f_{2}$ since it is not a point of $f$. Consequently $p$ must belong to $A_{2}$. Therefore $B_{1}<A_{2}$ and similarly $B_{2}<A_{1}$. It is now easily seen that the sets $A_{1}-A_{1} \cdot A_{2}$ and $A_{2}-A_{2} \cdot A_{1}$ are both included in $f$ and hence $A_{1}$ and $A_{2}$ differ by at most a set of points of measure zero. It follows from the absolute continuity of $\pi(A)$ that $\pi\left(A_{1}\right)=\pi\left(A_{2}\right)$. Hence $\pi^{\prime}\left(A^{\prime}\right)$ is uniquely determined for any set $A^{\prime}$ of $F^{\prime}$.

If $K$ is any collective with respect to the space $S^{\prime}$ and the distribution function $\pi^{\prime}\left(A^{\prime}\right)$, then $K$ is a collective with respect to the space $S$ and the 
distribution function $\pi(A)$, since if $e^{(n)}$ is any term of $K$, it will belong to the set $A$ if and only if it belongs to the corresponding set $A^{\prime}$.

We shall consider fields which are still further restricted. We shall decompose the space $S$ into $2^{\alpha}$ mutually exclusive hypercubes $m_{1,1}, m_{1,2}, \cdots, m_{1,2^{\alpha}}$. The hypercube $m_{1, j}$ is called a mesh and consists of the points whose coordinates $y_{1}, y_{2}, \cdots, y_{\alpha}$ satisfy the inequalities $a_{i, j} / 2 \leqq y_{i}<\left(a_{i, j}+1\right) / 2$ where each $a_{i, j}$ has the value 1 or 0 and $i=1,2, \cdots, \alpha$. Then $m_{1,1}+m_{1,2}+\cdots+m_{1,2}{ }^{\alpha}=S$. We shall designate as the lattice $L_{1}$, the field which consists of the null set and sums of the meshes $m_{1, j}$. We shall form a lattice $L_{2}$ by decomposing each of the meshes $m_{1, j}$ into $2^{\alpha}$ hypercubes (or meshes) in the same manner in which we decomposed the space $S$ to form the lattice $L_{1}$. Thus $L_{2}$ contains the meshes $m_{2,1}, m_{2,2}, \cdots, m_{2,2^{2 \alpha}}$. In a similar manner we shall form lattices $L_{3}, L_{4}, \cdots$. Thus the lattice $L_{s}$ contains $2^{s \alpha}$ mutually exclusive hypercubes or meshes. We shall assume that the subscripts of the meshes are so chosen that

$$
m_{s-1, j+1}=m_{s, j \cdot 2^{\alpha}+1}+m_{s, j \cdot 2^{\alpha}+2}+\cdots+m_{s,(j+1) 2^{\alpha}} .
$$

We shall let $N=L_{1}+L_{2}+\cdots$ and shall call $N$ a net. It is easily seen that $N$ is a field.

The point whose coordinates are $a_{1, j} / 2, a_{2, j} / 2, \cdots, a_{\alpha, j} / 2$ will be called the vertex of the mesh $m_{1, j}$. The vertices of the meshes of the lattices $L_{2}, L_{3}, \cdots$ will be located in corresponding positions. Thus for the case $\alpha=2$, a vertex is located in the lower left-hand corner of its mesh. Let $S^{\prime \prime}$ be the space which consists of all the vertices of the meshes of $N$. Then $S^{\prime \prime}$ is denumerable and everywhere dense in $S$. We shall define a field $F^{\prime \prime}$ of the space $S^{\prime \prime}$ as follows. A set $A^{\prime \prime}$ will be an element of $F^{\prime \prime}$ if and only if there exists an element $A$ of the net $N$ such that $A^{\prime \prime}=A \cdot S^{\prime \prime}$. The field $F^{\prime \prime}$ may be regarded as an approximation to the field $F$ since if $A$ is a set whose frontier points are of measure zero and if $\epsilon$ is any given positive number, there will exist two elements $A_{1}$ and $A_{2}$ of $N$ such that $A_{1}<A<A_{2}$ and $m\left(A_{2}-A_{1}\right)<\epsilon$.

11. Groups of operations. We shall obtain a more convenient form for conditions (C1), (C2), and (C3) as applied to the space $S^{\prime}$ and the field $F^{\prime}$ defined in $\$ 10$. Conditions (C2) and (C3) enable us to operate on a collective with any finite group of selections and contractions. The resultant of these operations is a collective and hence must satisfy (C1). Since the resultant of two contractions is a contraction and the resultant of two selections of $D$ is a selection of $D$ and since $D$ contains the identity selection and there exists an identity contraction, there will be no loss of generality in assuming that the selections and contractions of such a group alternate, beginning with

* Copeland II. 
a selection and ending with a contraction. Let the selections of such a group be denoted by $x_{1}, x_{2}, \cdots, x_{\sigma}$ and let the contractions be performed with respect to the sets $A_{1}, A_{2}, \cdots, A_{\sigma}$ where $A_{\sigma}<A_{\sigma-1}<\cdots<A_{2}<A_{1}$. Then the group is ordered as follows: $x_{1}, A_{1}, x_{2}, A_{2}, \cdots, x_{\sigma}, A_{\sigma}$.

Condition (C1) involves a group of selections $x_{r_{1}, n}, x_{r_{2}, n}, \cdots, x_{r_{h}, n}$ of the set $D_{0}$ and a group of sets $E_{1}, E_{2}, \cdots, E_{h}$. Let $\mu=\sigma+h$. Then conditions (C1), (C2), (C3) may be stated in terms of certain groups of operations involving $\mu$ selections and $\mu$ sets where $\mu=1,2,3, \cdots$.

The set $D+D_{0}$ is denumerable. Hence we can assume the existence of a one-to-one correspondence between the set of all non-negative integers $\lambda$ and the set of all ordered finite subsets of $D+D_{0}$. We shall demand that corresponding to each $\lambda$, the group of selections contains at least one from the set $D_{0}$. The space $S^{\prime}$ is denumerable and the field $F^{\prime}$ has the power of the continuum. Hence we can assume that a one-to-one correspondence has been established between the elements of $F^{\prime}$ and the set of numbers in the interval $0 \leqq \vartheta<1$. Let $t$ be any non-negative real number and let $\lambda=[t]$ be the largest integer contained in $t$. Then $t$ determines $\lambda$ and $\lambda$ determines a set of $\mu$ selections. Hence $t$ determines the integer $\mu$. Let $\vartheta=t-[t]$ be written in the binary scale. This number may be regarded as a sequence and hence can be operated upon by the selection $x_{1, \mu} \cdot{ }^{*}$ The sequence $\vartheta \subset x_{1, \mu}$ may in turn be regarded as a number in the interval from 0 to 1 . This number determines a set $A_{1}$. The numbers $\vartheta \subset x_{2, \mu}, \vartheta \subset x_{3, \mu}, \cdots, \vartheta \subset x_{\sigma, \mu}, \vartheta \subset x_{\sigma+1, \mu}, \cdots$, $\vartheta \subset x_{\mu, \mu}$ determine respectively the sets $A_{2}, A_{3}, \cdots, A_{\sigma}, E_{1}, E_{2}, \cdots, E_{h}$.

The group of operations thus performed on the collective can now be defined as follows. Let

$$
O_{i, t}(K)=O_{i-1, t}(K) \subset x_{i} \cdot \subset \cdot \phi_{A_{i}}\left[O_{i-1, t}(K) \subset x_{i}\right],
$$

where $0<i \leqq \sigma$ and $O_{0, t}(K)=K$. Let

$$
O_{t}(K)=\phi_{E_{1}}\left[O_{\sigma, t}(K) \subset x_{r_{1}, n}\right] \cdot \phi_{E_{2}}\left[O_{\sigma, t}(K) \subset x_{r_{2}, n}\right] \cdots \phi_{E_{h}}\left[O_{\sigma, t}(K) \subset x_{r_{h}, n}\right]
$$

and

$$
P[t, \pi(E)]=\pi\left(E_{1}\right) \cdot \pi\left(E_{2}\right) \cdots \pi\left(E_{h}\right) \cdot \pi^{-h}\left(A_{\sigma}\right) .
$$

Let $\Sigma^{\prime}$ be the set of points $t$ such that $A_{1}>A_{2}>\cdots>A_{\sigma}>E_{1}+E_{2}+\cdots$ $+E_{h}$ and $\pi\left(A_{\sigma}\right)>0$. Then conditions (C1), (C2), (C3) are equivalent to the following

$$
p\left[O_{t}(K)\right]=P[t, \pi(E)] \text { for every } t \text { belonging to } \Sigma^{\prime} .
$$

In the case of the space $S^{\prime \prime}$ and the field $F^{\prime \prime}$ defined in $\$ 10$, a similar set

* The ambiguous representation is resolved in the same manner as in the case of selections except that the number 0 is the sequence $0,0,0, \cdots$. 
of conditions can be obtained. Namely let $\Sigma^{\prime \prime}$ be the subset of $\Sigma^{\prime}$ for which $A_{1}, A_{2}, \cdots, A_{\sigma}, E_{1}, E_{2}, \cdots, E_{h}$ are elements of the field $F^{\prime \prime}$. Then for the field $F^{\prime \prime}$, conditions (C1), (C2), (C3) have the form

$$
p\left[O_{t}(K)\right]=P[t, \pi(E)] \text { for every } t \text { belonging to } \Sigma^{\prime \prime} .
$$

12. Existence of collectives. We shall prove the existence of collectives satisfying condition (C5). A one-dimensional label space $S$ will be chosen and the mesh $m_{s, j}$ will be the interval $2^{-s}(j-1) \leqq y<2^{-s} \cdot j$. The proof is accomplished by the aid of a new number scale. Let $y=\sum_{\nu=1}^{\infty} y^{(\nu)} \cdot 2^{-\nu(\nu+1) / 2}$ where $y^{(v)}=0,1,2, \cdots, 2^{v}-1$. Any number $y$ in the closed interval from 0 to 1 can be so developed. This number scale possesses the same type of ambiguity as is found in ordinary scales. Let $K(y)$ be the sequence

$$
K(y)=P^{(1)}(y), P^{(2)}(y), P^{(3)}(y), \cdots,
$$

where $P^{(\nu)}(y)=y^{(\nu)} \cdot 2^{-\nu}$. We shall show that for almost every $y$ of the interval from 0 to 1 , the corresponding sequence $K(y)$ satisfies condition (C5). The proof of this statement is accomplished by means of four lemmas.

The first lemma is concerned with the measure of a set

$$
E=E\left[P^{\left(n_{1}\right)}(y) \epsilon E_{1} \cdot P^{\left(n_{2}\right)}(y) \epsilon E_{2} \cdots P^{\left(n_{\nu}\right)}(y) \epsilon E_{\nu}\right]
$$

defined as follows. The expression $P^{\left(n_{i}\right)}(y) \epsilon E_{i}$ means that the point $P^{\left(n_{i}\right)}(y)$ is an element of the set $E_{i}$. The dots joining expressions of this type are conjunctive. The set $E$ consists of all points $y$ which satisfy the conjunction of all conditions within the square brackets. The set $E_{i}$ is the sum of $a_{i}$ meshes $m_{s, j}$ where $s$ is independent of $i$. Hence $m\left(E_{i}\right)=a_{i} 2^{-s}$.

LeMMA 1. $m\left\{E\left[P^{\left(n_{1}\right)}(y) \epsilon E_{1} \cdot P^{\left(n_{2}\right)}(y) \epsilon E_{2} \cdots P^{\left(n_{\nu}\right)}(y) \epsilon E_{\nu}\right]=m\left(E_{1}\right) \cdot m\left(E_{2}\right)\right.$ $\cdots m\left(E_{\nu}\right)$ if $s \leqq n_{1}<n_{2}<\cdots<n_{\nu}$.

The measure of the set of points $y$ for which the first $n_{\nu}$ digits are prescribed is $2^{-n_{\nu}\left(n_{\nu}+1\right) / 2}$. Thus the proof resolves itself into the problem of counting the number of possible combinations of the first $n_{\nu}$ digits of $y$ for which the conditions within the square brackets are satisfied. The first condition will be satisfied if $P^{\left(n_{1}\right)}(y)$ belongs to one of the $a_{1}$ meshes $m_{s, j}$. The point $P^{\left(n_{1}\right)}(y)$ will belong to the mesh $m_{s, j}$ if $2^{-s} \cdot(j-1) \leqq y^{\left(n_{1}\right)}<2^{-s} \cdot j$. Hence there are $2^{n_{1}-s}$ values for the digit $y^{\left(n_{1}\right)}$ consistent with the condition that $P^{\left(n_{1}\right)}(y)$ belongs to $m_{s, j}$. Therefore the number of values for this digit consistent with the first condition within the square brackets is $a_{1} \cdot 2^{n_{1}-s}$. The digits $y^{\left(n_{2}\right)}, y^{\left(n_{3}\right)}, \cdots, y^{\left(n_{\nu}\right)}$ can be similarly treated. There remain certain digits of $y$ which are entirely unrestricted. That is, the first digit of $y$ can be selected in $2^{1}$ ways, the second in $2^{2}$ ways, $\cdots$, the $\left(n_{1}-1\right)$ st in $2^{n_{1}-1}$ ways, the $\left(n_{1}+1\right)$ st in $2^{n_{1}+1}$ ways, $\cdots$. 
Thus these unrestricted digits can be selected in $2^{1+2+\cdots+n_{\nu}-n_{1}-n_{2}-\cdots-n_{\nu}}$ $=2^{n_{\nu}\left(n_{\nu}+1\right) / 2-n_{1}-n_{2}-\cdots-n_{\nu}}$ ways. It follows that

$$
\begin{aligned}
& m\left\{E\left[P^{\left(n_{1}\right)}(y) \epsilon E_{1} \cdot P^{\left(n_{2}\right)}(y) \epsilon E_{2} \cdots\right]\right\} \\
& =a_{1} 2^{n_{1}-s} a_{2} 2^{n_{2}-8} \cdots a_{\nu} 2^{n_{\nu}-8} 2^{n_{\nu}\left(n_{\nu}+1\right) / 2-n_{1}-n_{2}-\cdots-n_{\nu} 2^{-n_{\nu}\left(n_{\nu}+1\right) / 2}} \\
& =m\left(E_{1}\right) \cdot m\left(E_{2}\right) \cdots m\left(E_{\nu}\right) .
\end{aligned}
$$

The second lemma will be similar to the first except that it will be applied to the sequence $O_{\sigma, t}[K(y)]$ instead of to $K(y)$. We shall assume that the sets $A_{1}, A_{2}, \cdots, A_{\sigma}$ used in defining the operator $O_{\sigma, t}$ and the sets $E_{1}, E_{2}, \cdots, E_{\nu}$ used in the statement of the lemma are all sums of meshes $m_{s, j}$ with the same subscript $s$. Let

$$
O_{i, t}[K(y)]=P_{i}^{(1)}(y), P_{i}^{(2)}(y), \cdots .
$$

LEMMA 2. $m\left\{E\left[P_{\sigma}^{\left(n_{1}\right)}(y) \epsilon E_{1} \cdot P_{\sigma}^{\left(n_{2}\right)}(y) \epsilon E_{2} \cdots\right]\right\}=m\left(E_{1}\right) \cdot m\left(E_{2}\right) \cdots m\left(E_{v}\right)$ $\cdot m^{-\nu}\left(A_{\sigma}\right)$ if $s \leqq n_{1}<n_{2}<\cdots<n_{\nu}$.

We shall prove the lemma inductively. That is, we shall prove that if the equation is true when $\sigma$ is replaced by $i-1$, it will then be true when $\sigma$ is replaced by $i$. If $\sigma=0$, then the above equation reduces to the corresponding equation of Lemma 1 , since $m^{-v}(S)=1$. The sequence $O_{i, t}[K(y)]$ is obtained from the sequence $O_{i-1, t}[K(y)]$ by the selection $x_{i}$ and the contraction with respect to the set $A_{i}$. Both of these operations throw away certain terms of the sequence $O_{i-1, t}[K(y)]$. The terms thrown away by the selection are entirely unrestricted. However any term thrown away by the contraction must belong to the set $A_{i-1}$ but not to the set $A_{i}$. On the basis of the assumption that the lemma is true when $\sigma$ is replaced by $i-1$, it follows that the measure of the set of points for which the $n_{\nu}$ th term of $O_{i, t}[K(y)]$ correponds to the $\left(n_{\nu}+\mu\right)$ th term of the sequence $O_{i-1, t}[K(y)] \subset x_{i}$ and for which $P^{\left(n_{1}\right)}(y) \epsilon E_{1} \cdot P^{\left(n_{2}\right)}(y) \epsilon E_{2} \cdots P^{\left(n_{\nu}\right)}(y) \epsilon E_{\nu}$ is $m\left(E_{1}\right) \cdot m\left(E_{2}\right) \cdots m\left(E_{\nu}\right)$ - $m^{n_{\nu}-\nu}\left(A_{i}\right)\left[m\left(A_{i-1}\right)-m\left(A_{i}\right)\right]^{\mu} \cdot m^{-n_{\nu}-\mu}\left(A_{i-1}\right)$. That is, a certain $\nu$ of the terms of $O_{i-1, t}[K(y)]$ must belong respectively to the sets $E_{1}, E_{2}, \cdots, E_{\nu}$ and $n_{\nu}-\nu$ of the terms must belong to $A_{i}$ while the remaining $\mu$ terms must belong to $A_{i-1}$ but not to $A_{i}$. The $\left(n_{\nu}+\mu\right)$ th term must belong to $E_{\nu}$. Since $\mu$ can take on all values from 0 to $\infty$ and since there are ${ }_{n_{\nu}+\mu-1} C_{\mu}$ ways in which the $\mu$ terms can be selected, then

$$
\begin{aligned}
& m\left\{E\left[P_{i}^{\left(n_{1}\right)}(y) \epsilon E_{1} \cdot P_{i}^{\left(n_{2}\right)}(y) \epsilon E_{2} \cdots\right]\right\} \\
& =m\left(E_{1}\right) \cdot m\left(E_{2}\right) \cdots m\left(E_{\nu}\right) \cdot m^{n_{\nu}-\nu}\left(A_{i}\right) m^{-n_{\nu}}\left(A_{i-1}\right) \cdot \sum_{\mu=0}^{\infty}{ }_{n_{\nu}+\mu-1} C_{\mu} \\
& \cdot\left[m\left(A_{i-1}\right)-m\left(A_{i}\right)\right]^{\mu} \cdot m^{-\mu}\left(A_{i-1}\right)
\end{aligned}
$$




$$
\begin{aligned}
= & m\left(E_{1}\right) \cdot m\left(E_{2}\right) \cdots m\left(E_{\nu}\right) \cdot m^{-\nu}\left(A_{i}\right) \cdot m^{n_{\nu}}\left(A_{i}\right) \cdot m^{-n_{\nu}}\left(A_{i-1}\right) \\
& \cdot\left[1-\frac{m\left(A_{i-1}\right)-m\left(A_{i}\right)}{m\left(A_{i-1}\right)}\right]^{-n_{\nu}} \\
= & m\left(E_{1}\right) \cdot m\left(E_{2}\right) \cdots m\left(E_{\nu}\right) \cdot m^{-\nu}\left(A_{i}\right) .
\end{aligned}
$$

It follows inductively that this equation still holds when $i=\sigma$.

It will be observed that the operator $x_{s, 1}$ selects the $s$ th term and all of the succeeding terms of a sequence $K$. If the expression $K(y)$ in Lemma 2 is replaced by $K^{\prime}(y)=K(y) \subset x_{s, 1}$, then the condition $s \leqq n_{1}$ will no longer be necessary.

\section{LEMMA 3. The function}

$$
V^{\prime}(y, t)=\limsup _{\mu \rightarrow \infty}\left|p_{\mu}\left\{O_{t}\left[K^{\prime}(y)\right]\right\}-P[t, m(E)]\right|
$$

is equal to 0 almost everywhere in the interval $\Delta: 0<y<1$.

If we let $E\left[V^{\prime}(y, t)>\epsilon\right]$ be the set of points for which $V^{\prime}(y, t)>\epsilon$, then

$$
E\left(V^{\prime}>0\right)=E\left(V^{\prime}>1 / 2\right)+E\left(V^{\prime}>1 / 3\right)+E\left(V^{\prime}>1 / 4\right)+\cdots
$$

Hence the lemma will be established if we can prove that $m\left[E\left(V^{\prime}>\epsilon\right)\right]=0$ for every positive number $\epsilon$. We have the equation

$$
E\left(V^{\prime}>\epsilon\right)=\lim _{\mu_{0} \rightarrow \infty} \sum_{\mu=\mu_{0}}^{\infty} E\left(\left|p_{\mu}\left\{O_{t}\left[K^{\prime}(y)\right]\right\}-P[t, m(E)]\right|>\epsilon\right),
$$

and hence

$$
m\left[E\left(V^{\prime}>\epsilon\right)\right] \leqq \sum_{\mu=\mu_{0}}^{\infty} m\left[E\left(\left|p_{\mu}\left\{O_{t}\left[K^{\prime}(y)\right]\right\}-P[t, m(E)]\right|>\epsilon\right)\right]
$$

for every positive integer $\mu_{0}$. The remainder of the proof consists in establishing the convergence of this series. From Lemma 2 it follows that

$$
\begin{aligned}
& m\left[E\left(\left|p_{\mu}\left\{O_{t}\left[K^{\prime}(y)\right]\right\}-P[t, m(E)]\right|>\epsilon\right)\right]=\sum_{|\varepsilon / \mu-P[t, m(E)]|>\epsilon}{ }_{\mu} C_{s} \\
& \cdot P^{s}[t, m(E)]\{1-P[t, m(E)]\}^{\mu-s},
\end{aligned}
$$

where the expression below the summation sign indicates that $s$ is summed over all values consistent with this inequality. Borel has proved the convergence of all series of the form*

$$
\sum_{\mu=1}^{\infty} \sum_{\left\{s / \mu-p \mid>{ }_{e}\right.} C_{s} \cdot p^{s} \cdot q^{\mu-s},
$$

* Borel I. 
where $p$ and $q$ are positive numbers and $p+q=1$. Hence Lemma 3 is established.

The purpose of Lemma 4 is to show that Lemma 3 will still be true if $K^{\prime}(y)$ is replaced by $K(y)$. We shall first construct a set $D^{\prime}$ defined as follows. If $x$ is any selection of $D$ and if $s$ is any positive integer, then there will exist a selection $x^{\prime}$ of $D^{\prime}$ such that

$$
K \subset x \cdot \subset \cdot x_{s^{\prime}, 1}=K \subset x_{s, 1} \cdot \subset \cdot x^{\prime},
$$

where $s^{\prime}$ is such that either $n_{s^{\prime}-1}<s \leqq n_{s^{\prime}}$ or $s \leqq n_{1}$, and where $n_{1}, n_{2}, n_{3}, \cdots$ are the ordinal numbers of the terms selected by $x$. Thus $n_{s^{\prime}}-s+1$, $n_{s^{\prime}+1}-s+1, \cdots$ are the ordinal numbers of the terms selected by $x^{\prime}$. Since the selection $x_{1,1}$ is the identity, it follows that $D<D^{\prime}$. Moreover $D^{\prime}$ is denumerable. In Lemma 4 it will be assumed that the set $\Sigma^{\prime \prime}$ is defined in terms of $D^{\prime}$.

LEMMA 4. If $t$ is any number belonging to the set $\Sigma^{\prime \prime}$ and if $s$ is any positive integer, then there exists a number $t^{\prime}$ belonging to $\Sigma^{\prime \prime}$ and a positive integer $r$ such that

$$
O_{t}(K) \subset x_{r, 1}=O_{t^{\prime}}\left(K \subset x_{s, 1}\right) .
$$

Given the selection $x$ and the positive integer $s$, there exist a selection $x^{\prime}$ and a positive integer $s^{\prime}$ such that $K \subset x \cdot \subset \cdot x_{s^{\prime}, 1}=K \subset x_{s, 1} \cdot \subset \cdot x^{\prime}$. There exists a second positive integer $s^{\prime \prime}$ such that $K \subset x \cdot \subset \cdot x_{s^{\prime}, 1}: \subset: \phi_{E}\left(K \subset x \cdot \subset \cdot x_{s^{\prime}, 1}\right)$ $=K \subset x \cdot \subset \cdot \phi_{E}(K \subset x): \subset: x_{s^{\prime \prime}, 1}$. From this it follows inductively that there exist a positive integer $r$ and a number $t^{\prime}$ belonging to $\Sigma^{\prime \prime}$ such that

$$
O_{\sigma, t}(K) \subset x_{r, 1}=O_{\sigma, t^{\prime}}\left(K \subset x_{8,1}\right) \text {. }
$$

The number $t^{\prime}$ differs from $t$ because the selections involved in the operator $O_{\sigma, t^{\prime}}$ are different from those involved in the operator $O_{\sigma, t}$. The contractions are the same for both operators. Since

$$
\phi_{E}\left[O_{\sigma, t}(K) \subset x_{r, 1}\right]=\phi_{E}\left[O_{\sigma, t}(K)\right] \subset x_{r, 1},
$$

we have the equation

$$
O_{t}(K) \subset x_{r, 1}=O_{t^{\prime}}\left(K \subset x_{s, 1}\right) .
$$

With the aid of Lemmas 1, 2, 3, 4, we can prove the following theorem.

THEOREM 1. For almost every y of the interval $\Delta: 0<y<1$, the corresponding sequence $K(y)$ satisfies condition (C5).

Let

$$
V^{\prime}\left(y, t^{\prime}\right)=\limsup _{\mu \rightarrow \infty}\left|p_{\mu}\left\{O_{t^{\prime}}\left[K(y) \subset x_{s, 1}\right]\right\}-P[t, m(E)]\right|
$$




$$
V(y, t)=\limsup _{\mu \rightarrow \infty}\left|p_{\mu}\left\{O_{t}[K(y)]\right\}-P[t, m(E)]\right| .
$$

The sets involved in the operator $O_{t^{\prime}}$ are sums of meshes $m_{s, j}$. Of course for any given operator $O_{t^{\prime}}$, it is only necessary to let $s$ be the subscript of the finest mesh occurring in the sets corresponding to this operator. Since the contractions involved in $O_{t^{\prime}}$ are the same as those involved in $O_{t}, s$ depends directly upon $t$. The number $t^{\prime}$ depends upon both $t$ and $y$. Let $M$ be the set of points $y$ for which $V^{\prime}\left(y, t^{\prime}\right)=0$ for every $t^{\prime}$ of the set $\Sigma^{\prime \prime}$. Since $\Sigma^{\prime \prime}$ is denumerable, it follows from Lemma 3 that $m(M)=1$. Let $y$ be any point of $M$ and $t$ any point of $\Sigma^{\prime \prime}$. Then by Lemma 4 there exists a pair of numbers $r$ and $t^{\prime}$ such that

$$
p\left\{O_{t^{\prime}}\left[K(y) \subset x_{s, 1}\right]\right\}=p\left\{O_{t}[K(y)] \subset x_{r, 1}\right\}=p\left\{O_{t}[K(y)]\right\} .
$$

And hence $V(y, t)=V^{\prime}\left(y, t^{\prime}\right)=0$. Therefore for almost every $y$, the corresponding sequence $K(y)$ satisfies condition (C5).

13. Segments of collectives. We shall now consider the problem of constructing collectives satisfying condition (C4). The method will appear considerably different from that of the previous problem but will in fact be guided by it. A study of the differences between these two problems will be of advantage in the work which we shall do. We now have to deal with sets whose frontier points are of measure zero instead of with sets consisting of finite sums of meshes. The collective $K(y)$ is so constructed that each term is capable of adapting itself to a finer mesh than is the preceding term. That is, the point $P^{(s)}(y)$ can belong to any one of the meshes of the lattice $L_{s}$ whereas the point $P^{(s-1)}(y)$ cannot. Thus after a certain term in this collective all points are capable of adapting themselves to any given set consisting of a finite sum of meshes. However if we have a set whose frontier points are of measure zero, then in general the adaptation is never complete. Moreover the tendency toward adaptation is so rapid that it is difficult to follow the variation of the success ratio. Our first point of difference from the preceding method will be for the purpose of slowing up this adaptation.

We shall generalize the method of formation of the sequence $K(y)$. Let $N_{1}, N_{2}, N_{3}, \cdots$ be a sequence of positive integers which will be determined at a later point. Let $\nu_{s}=N_{1}+N_{2}+\cdots+N_{s-1}$ if $1<s$ and $\nu_{1}=0$. Let $P^{(k)}(y)=\left[y^{(k)} \cdot 2^{s-k}\right] \cdot 2^{-s}$ where $s$ is determined so that $\nu_{s}<k \leqq \nu_{s+1}$. It will be observed that if $N_{1}=N_{2}=\cdots=1$, then $k=s$ and this definition of $K(y)$ is the same as the previous one. We shall say that the points $P^{\left(\nu_{s}+1\right)}(y), P^{\left(\nu_{s}+2\right)}(y), \cdots, P^{\left(\nu_{s}+1\right)}(y)$ constitute the sth segment of $K(y)$. These points are adapted to the meshes $m_{s, j}$. The sequences $O_{i, t}[K(y)]$ admit of a similar decomposition into segments. Let 


$$
\begin{aligned}
\mu(s, i, y, t) & =\nu(s, i-1, y, t) \cdot p_{\nu(s, i-1, y, t)}\left(x_{i}\right), \\
\nu(s, i, y, t) & =\mu(s, i, y, t) \cdot p_{\mu(s, i, y, t)}\left(\phi_{A_{i}}\left\{O_{i-1, t}[K(y)] \subset x_{i}\right\}\right),
\end{aligned}
$$

where $\nu(s, 0, y, t)=\nu_{s}$. The points of $O_{i, t}[K(y)]$ whose superscripts range from $\nu(s, i, y, t)+1$ to $\nu(s+1, i, y, t)$ constitute the sth segment of $O_{i, t}[K(y)]$. If $N(s, i, y, t)=\nu(s+1, i, y, t)-\nu(s, i, y, t)$, then there are $N(s, i, y, t)$ points in the sth segment of this sequence. The points are selected from the sth segment of $K(y)$. Let

$$
\nu(s, \sigma, y, t) \cdot p_{\nu(s, \sigma, y, t)}\left(x_{r_{h}, n}\right)=\mu(s, y, t) .
$$

Then

$$
r_{h}+n \cdot[\mu(s, y, t)-1] \leqq \nu(s, \sigma, y, t)<r_{h}+n \cdot \mu(s, y, t) .
$$

The points of $O_{t}[K(y)]$ whose superscripts range from $\mu(s, y, t)+1$ to $\mu(s+1, y, t)$ constitute the $s$ th segment of this sequence. All except the first of the points of this segment correspond to points of the sth segment of $K(y)$. The first point of the sth segment of $O_{t}[K(y)]$ corresponds to $n$ points which may be selected from both the $(s-1)$ st and the sth segments of $K(y)$. Let $J(s, y, t)=\mu(s+1, y, t)-\mu(s, y, t)$. Then there are $J(s, y, t)$ points in the $s$ th segment of $O_{t}[K(y)]$. It will be observed that $[N(s, \sigma, y, t) / n] \leqq J(s, y, t)$. We shall investigate the success ratio of the sequence $O_{t}[K(y)]$ within its $s$ th segment, that is, the expression $p_{N}\left\{O_{t}[K(y)] \subset x_{\mu, 1}\right\}$ where $\mu=\mu(s, y, t)$ and $0<N \leqq J(s, y, t)$. Our problem is to choose the integers $N_{s}$ so that this success ratio can be made to approximate $P[t, m(E)]$ for a set of points $y$ whose measure approximates 1 .

14. Lengths of segments. The sth segment of $O_{t}[K(y)]$ must contain a sufficient number of terms to permit the success ratio within this sequence to approximate the desired value. In order to accomplish this, there must also be lower bounds to the lengths of the sth segments of the sequences $O_{i, t}[K(y)]$. We shall appraise the measures of certain sets of points $y$ for which these segments have the desired lengths. Thus the lower bounds should be numbers which are independent of $y$. Let

$$
M(s, i, t)=\min N(s, i-1, t) \cdot p_{N(s, i-1, t)}\left(x_{i} \subset x_{\nu, 1}\right),
$$

where $\nu=\nu(s, i-1, y, t)$ and where the minimum is taken with respect to $y$;

$$
N(s, i, t)=\left[M(s, i, t) \cdot\left\{m\left(A_{s, i}\right) / m\left(A_{s, i-1}\right)-2^{-s-1}\right\}\right],
$$

where the square brackets signify the largest integer contained in the number within these brackets;

$$
N(s, 0, t)=N_{s} .
$$


The numbers $N(s, i, t)$ thus inductively defined will be taken as the lower bounds of the lengths $N(s, i, y, t)$ of the sth segments of the sequences $O_{i, t}[K(y)]$. We shall let $G(s, i, t)=E[N(s, i, t) \leqq N(s, i, y, t)]$, where $0<i \leqq \sigma$, denote the set of points $y$ for which this segment has the desired length. Since the integers $\nu=\nu(s, i-1, y, t)$ depend only on the first $\nu_{s}$ digits of $y$, it is easily seen that $\lim _{N_{s} \rightarrow \infty} N(s, i, t)=\infty$, and hence $N(s, i, t)$ can be made arbitrarily large by a proper choice of $N_{s}$. Let

$$
J(s, t)=[N(s, \sigma, t) / n] .
$$

Then the length $J(s, y, t)$ of the sth segment of $O_{t}[K(y)]$ will be at least as great as $J(s, t)$ for all values of $y$ for which $N(s, \sigma, t) \leqq N(s, \sigma, y, t)$. Let

$$
H(s, t)=\left[J(s, t) \cdot 2^{-8-1}-\mu(s, t)-\nu_{s}\right],
$$

where $\mu(s, t)=J(1, t)+J(2, t)+\cdots+J(s-1, t)$. Then $\lim _{N_{s} \rightarrow \infty} H(s, t)=\infty$. Let

$$
E_{s, i}=\sum_{2^{-8}(j-1) \epsilon E_{i}} m_{s, j} \text { and } A_{s, i}=\sum_{2^{-s}(j-1) \epsilon A_{i}} m_{s, j} .
$$

Thus $E_{s, j}$ is a sum of meshes of the lattice $L_{s}$. It consists of all such meshes whose vertices lie in $E_{j}$. The set $A_{s, j}$ is similarly defined. Let

$$
\begin{array}{r}
P[s, t, m(E)]=m\left(E_{s, 1}\right) \cdot m\left(E_{s, 2}\right) \cdots m\left(E_{s, h}\right) \cdot m^{-h}\left(A_{s, \sigma}\right), \\
G(s, t)=E\left(\left|p_{N}\left\{O_{t}[K(y)] \subset x_{\mu, 1}\right\}-P[s, t, m(E)]\right|<2^{-s-1} \cdot \subset \cdot H(s-1, t)\right. \\
\leqq N \leqq J(s, y)),
\end{array}
$$

where $\mu=\mu(s, y, t)$. The symbol $\subset$ means "if." The set $G(s, t)$ consists of those points $y$ for which the inequality to the left of the symbol $\subset$ holds for all values of $N$ satisfying the inequalities to the right of this symbol. Thus if $y$ is a member of this set, then a restriction is placed upon the success ratio of $O_{t}[K(y)]$ within its $s$ th segment.

The integers $N(s, i, t), J(s, t), H(s, t)$ depend upon the numbers $N_{s}$. While the determination of the numbers $N_{s}$ will not be given until a later point, it is necessary to note here that the numbers $N(s, i, t), J(s, t), H(s, t)$ must all be positive. It then follows from the definition of $H(s, t)$ that $\{H(s, t)$ $+\mu(s, t)\} \cdot 2^{s+1} \leqq J(s, t), J(s-1, t)<J(s, t)$ and $H(s-1, t) \leqq H(s, t)<J(s, t)$. Hence $H(s-1, t)<J(s, y, t)$ whenever $N(s, \sigma, t) \leqq N(s, \sigma, y, t)$.

15. Appraisals of certain set measures. We shall prove that

$$
\begin{aligned}
m\{C[G(s, 1, t) \cdot G(s, 2, t) & \cdots G(s, \sigma, t)]\} \\
& \leqq \alpha(s, 1, t)+\alpha(s, 2, t)+\cdots+\alpha(s, \sigma, t)
\end{aligned}
$$

and 


$$
\begin{aligned}
m\{C[G(s, 1, t) \cdot G(s, 2, t) \cdots G(s, \sigma, t) \cdot G(s, t)]\} & \\
& \leqq \alpha(s, 1, t)+\alpha(s, 2, t)+\cdots+\alpha(s, \sigma, t)+\alpha(s, t),
\end{aligned}
$$

where

$$
\begin{aligned}
& \alpha(s, i, t)=_{R / M(s, i, t)<m\left(A_{s, i}\right) / m\left(A_{s, i-1}\right)-2^{-s-1}} \sum_{(s, i, t)} C_{R} \\
& \cdot\left(\frac{m\left(A_{s, i}\right)}{m\left(A_{s, i-1}\right)}\right)^{R}\left(1-\frac{m\left(A_{s, i}\right)}{m\left(A_{s, i-1}\right)}\right)^{M(s, i, t)-R},
\end{aligned}
$$

and

$$
\begin{aligned}
\alpha(s, t)=\sum_{N=H(s-1, t)}^{\infty} \sum_{|R / N-P[s, t, m(E)]| \geqq 2^{-s-1}-1 / N} \\
\cdot P^{R}[s, t, m(E)]\{1-P[s, t, m(E)]\}^{N-R} .
\end{aligned}
$$

The proof will be accomplished by means of the following lemmas.

LEMMA 1. $m\left\{E\left[P^{\left(n_{1}\right)}(y) \epsilon E_{1} \cdot P^{\left(n_{2}\right)}(y) \epsilon E_{2} \cdots P^{\left(n_{h}\right)}(y) \epsilon E_{h}\right]\right\}=m\left(E_{1}\right) \cdot m\left(E_{2}\right)$ $\cdots m\left(E_{h}\right)$, where $\nu_{s}<n_{1}<n_{2}<\cdots<n_{h}$ and where the sets $E_{1}, E_{2}, \cdots, E_{h}$ are elements of $L_{s}$.

LEMMA 2. $m\left\{E\left[P_{i}^{\left(n_{1}\right)}(y) \epsilon E_{1} \cdot P_{i}^{\left(n_{2}\right)}(y) \epsilon E_{2} \cdots\right]\right\} \leqq m\left(E_{1}\right) \cdot m\left(E_{2}\right) \cdots m\left(E_{h}\right)$ $\cdot m^{-h}\left(A_{s, i}\right)$, where $n_{1}=\nu(s, i, y, t)+\lambda_{1}<n_{2}=\nu(s, i, y, t)+\lambda_{2}<\cdots<n_{h}$ $=\nu(s, i, y, t)+\lambda_{h} \leqq \nu(s+1, i, y, t)$, where $\lambda_{1}, \lambda_{2}, \cdots, \lambda_{h}$ are positive integers independent of $y$ and $O_{i, t}[K(y)]=P_{i}{ }^{(1)}(y), P_{i}{ }^{(2)}(y), P_{i}{ }^{(3)}(y), \cdots$ and the sets $E_{1}, E_{2}, \cdots, E_{h}$ are elements of $L_{s}$.

The first lemma is proved in a manner similar to that of Lemma $1, \S 12$. In order to prove Lemma 2, let us first consider a special case in which $t$ is restricted in such a manner that $A_{1}=A_{s, 1}, A_{2}=A_{s, 2}, \cdots, A_{i}=A_{s, i}$. For such a choice of $t$, it follows that the expressions to the left and to the right of the sign $\leqq$ would actually be equal if the restriction $n_{h} \leqq \nu(s+1, i, y, t)$ were removed. This fact can be proved in a manner similar to that of Lemma $2, \S 12$. The restriction $n_{h} \leqq \nu(s+1, i, y, t)$ makes the above specialization of $t$ unnecessary. Moreover this restriction in general decreases the set whose measure we are appraising. Hence Lemma 2 is established.

We have the equation

$$
\begin{aligned}
m\{C[G(s, 1, t) \cdot G(s, 2, t) \cdots G(s, i, t)]\} & \\
= & m\{C[G(s, 1, t) \cdots G(s, i-1, t)]\} \\
& +m\{G(s, 1, t) \cdots G(s, i-1, t) C[G(s, i, t)]\} .
\end{aligned}
$$

We shall show that the measure of the $\operatorname{set} G(s, 1, t) \cdots G(s, i-1, t) C[G(s, i, t)]$ is less than or equal to $\alpha(s, i, t)$. If $y$ is a member of this set, then there must 
be at least $N(s, i-1, t)$ points in the $s$ th segment of $O_{i-1, t}[K(y)]$. Hence there must be at least $M(s, i, t)$ points in the sth segment of $O_{i-1, t}[K(y)] \subset x_{i}$. Suppose that $R$ of the first $N(s, i, t)$ points of the sth segment of $O_{i-1, t}[K(y)] \subset x_{i}$ belong to the set $A_{s, i}$. Then it must at least be true that $R<N(s, i, t)$ and hence $R / M(s, i, t)<m\left(A_{s, i}\right) / m\left(A_{s, i-1}\right)-2^{-s-1}$. It follows from Lemma 2 that $m\{G(s, 1, t) \cdot G(s, 2, t) \cdots G(s, i-1, t) \cdot C[G(s, i, t)]\} \leqq \alpha(s, i, t)$. Thus inequality (6) is established. In a like manner it is easily seen that inequality (7) is also true.

We are now in a position to determine the integers $N_{s}$. It will be observed that there are only a finite number of sets of the sets $A_{s^{\prime}, i}$ and $E_{s^{\prime}, i}$ such that $s^{\prime} \leqq s$ and $t \leqq s$, and only a finite number of sets of selections $x_{i}$ and $x_{r, n}$ such that $t \leqq s$. There correspond a finite set of values of $t$, the particular choice of the set being unimportant. By a proper selection of the integer $N_{s}$, the integers $M(s, i, t)$ can be made so large that the numbers $\alpha(s, i, t)$ can be made arbitrarily small for all values of $t$. Moreover $H(s-1, t)$ can be made so large that $\alpha(s, t)$ can be made arbitrarily small. We shall choose $N_{s}$ so that

$$
\sum_{(t)}\{\alpha(s, 1, t)+\alpha(s, 2, t)+\cdots+\alpha(s, \sigma, t)\} \leqq 2^{-8-1},
$$

where the summation is extended over all values of $t$ of the above mentioned finite set. Then

$$
m\left\{C\left(\prod_{(t)} G(s, 1, t) \cdot G(s, 2, t) \cdots G(s, \sigma, t)\right)\right\} \leqq 2^{-s-1},
$$

where the symbol $\prod_{(t)}$ means that the conditions to the right of this symbol must be satisfied simultaneously for all values of $t$. Let

$$
\prod_{s=s_{0}}^{\infty} \prod_{(t)} G(s, 1, t) \cdot G(s, 2, t) \cdots G(s, \sigma, t) \cdot G(s, t)=G_{s_{0}} .
$$

Then $m\left[C\left(G_{s_{0}}\right)\right] \leqq 2^{-s_{0}}$. Let

Then $m(G)=1$.

$$
G=\sum_{s_{0}=1}^{\infty} G_{s_{0}}
$$

We shall show that if $y$ is any member of the set $G$, then $K(y)$ satisfies condition (C4). This will be done with the aid of the following lemma.*

Lemma 3. If $K=P^{(1)}, P^{(2)}, P^{(3)}, \cdots$ is a sequence whose label space consists of the elements 0 and 1 and if there exist a decreasing sequence of positive

* This lemma was proved by Regan (see Regan I). A much simpler lemma whose function is similar has been proved by von Mises (von Mises VI). However I have been unable to adapt von Mises' lemma to this problem. 
numbers $\epsilon_{1}, \epsilon_{2}, \epsilon_{3}, \cdots$ such that $\lim _{s \rightarrow \infty} \epsilon_{s}=0$, and two sets of positive integers $J_{1}, J_{2}, J_{3}, \cdots$ and $H_{1}, H_{2}, H_{3}, \cdots$ such that for $s_{0} \leqq s$

(a)

$$
\left|p_{N}\left(K \subset x_{\mu, 1}\right)-p\right|<\epsilon_{s} / 3
$$

whenever $H_{s} \leqq N \leqq J_{s+1}$, where $\mu=\mu_{s+1}=J_{1}+J_{2}+\cdots+J_{s}$,

$$
\left|p_{J_{s}}\left(K \subset x_{\mu, 1}\right)-p\right|+\left(\mu_{s}+H_{s}\right) / J_{s}<\epsilon_{s} / 3 \text {, }
$$

where $\mu=\mu_{s}$, then

(c)

$$
\left|p_{N}(K)-p\right|<\epsilon_{s}
$$

if $\mu_{s+1} \leqq N \leqq \mu_{s+2}$ and $s_{0} \leqq s$.

Since $\lim _{s \rightarrow \infty} P[s, t, m(E)]=P[t, m(E)]$, it follows that there exists a decreasing sequence of positive numbers $\epsilon_{1}, \epsilon_{2}, \epsilon_{3}, \cdots$ such that

$$
|P[s, t, m(E)]-P[t, m(E)]|+2^{-s}<\epsilon_{s} / 3
$$

and $\lim _{s \rightarrow \infty} \epsilon_{s}=0$. From the definition of $H(s, t)$ it follows that $H(s, t)+\nu_{s}$ $\leqq J(s, t) \cdot 2^{-8-1}$. Hence

$$
[H(s, t)+\mu(s, y, t)] / J(s, y, t) \leqq\left[H(s, t)+\nu_{s}\right] / J(s, t) \leqq 2^{-s-1} .
$$

Let $y$ be a member of $G(s, t)$. If the inequality imposed by this condition be combined with (10) and (11), we get

$$
\left|p_{J(s, y, t)}\left\{O_{t}[K(y)] \subset x_{\mu, 1}\right\}-P[t, m(E)]\right|+[H(s, t)+\mu] / J(s, t) \leqq \epsilon_{s} / 3,
$$

where $\mu=\mu(s, y, t)$. In a like manner it can be proved that

$$
\left|p_{N}\left\{O_{t}[K(y)] \subset x_{\mu, 1}\right\}-P[t, m(E)]\right|<\epsilon_{s} / 3
$$

if $H(s, t) \leqq N \leqq J(s+1, y, t)$ and $\mu=\mu(s+1, y, t)$ and $y \epsilon G(s+1, t)$. Hence from Lemma 3 we conclude that

$$
\left|p_{N}\left\{O_{t}[K \cdot(y)]\right\}-P[t, m(E)]\right|<\epsilon_{s}
$$

if $\mu(s+1, y, t) \leqq N \leqq \mu(s+2, y, t)$ and if $y$ is an element of $G(s, t) \cdot G(s+1, t)$. If $y$ is an element of $G$, then there will exist a number $s_{0}$ such that condition (14) will hold whenever $s_{0} \leqq s$. This gives us the following theorem.

THEOREM 2. For almost every $y$ the corresponding sequence $K(y)$ satisfies condition (C4).

16. Generalizations. We shall consider the case in which the space $S$ is the region $0 \leqq y_{i}<1$, where $i=1,2,3, \cdots, \alpha$. Let $\pi(E)$ be an absolutely additive, absolutely continuous function defined in $S$ and such that $0<\pi(E)$ whenever $0<M(E)$. We shall construct a collective $K=P^{(1)}, P^{(2)}, P^{(3)}, \ldots$ which satisfies (C4). In order to do this, we shall set up a correspondence 
between the points of $S$ and the points of the interval $I: 0 \leqq y<1$. Let $S$ be decomposed into a system of meshes $m_{s, j}$ and let us define a corresponding set of intervals $I_{s, j}$. Let

$$
I_{s, j}: \quad \pi\left(m_{s, 1}+m_{s, 2}+\cdots+m_{s, j-1}\right) \leqq y<\pi\left(m_{s, 1}+\cdots+m_{s, j}\right),
$$

where $i<j$,

$$
I_{s, 1}: \quad 0 \leqq y<\pi\left(m_{s, 1}\right) .
$$

Corresponding to any $y$ of $I$ and to any positive integer $s$ there exists a positive integer $j_{s}$ such that $y$ is an element of $I_{s, j_{s}}$. Let

$$
P_{y}=\prod_{s=1}^{\infty} \bar{m}_{s, j_{s}}
$$

where $\bar{m}_{s, j_{8}}$ is the set consisting of the mesh $m_{s, j_{s}}$ plus the frontier points of this mesh. Thus corresponding to any point $y$ of the interval $I$ there is determined uniquely by this enclosure sequence, a point $P_{y}$ of the region $\bar{S}$, where $\bar{S}$ is equal to $S$ plus the frontier points of $S$. If $y$ is any point of an interval $I_{s, j}$, then $P_{y}$ is a point of the corresponding set $\bar{m}_{s, j}$. The inverse of this correspondence is not necessarily unique.

Let $P_{s, j}$ be the point of $S$ which corresponds to the point $2^{-8}(j-1)$ of the interval $I$. Let $A$ be any subset of $S$ having frontier points of measure zero. Let $s$ be any positive integer and $j_{1}, j_{2}, \cdots, j_{n}$ be the set of all positive integers such that the corresponding points $P_{s, j_{i}}$ are elements of the set $A$. It may of course happen that the points $P_{s, j_{1}}, P_{s, j_{2}}, \cdots, P_{s, j_{n}}$ of the set $A$ are not all distinct. Let $A_{s}^{\prime}=m_{s, j_{1}}^{\prime}+m_{s, j_{2}}^{\prime}+\cdots+m_{s, j_{n}}^{\prime}$, where the sets $m_{s, j}^{\prime}$ are meshes of the interval $I$.

We shall prove that $\lim _{s \rightarrow \infty} m\left(A_{s}{ }^{\prime}\right)=\pi(A)$. We shall prove this fact first for the case in which the set $A$ is one of the meshes $m_{\sigma, i}$. Given any positive number $\epsilon$ there exists a positive number $\sigma^{\prime}$ and a corresponding element $B_{\sigma^{\prime}}$ of $L_{\sigma^{\prime}}$ such that all of the points which belong to $m_{\sigma, i}$ are interior points of $B_{\sigma^{\prime}}$, and $\pi\left(B_{\sigma^{\prime}}\right)<\pi\left(m_{\sigma, i}\right)+\epsilon$. Corresponding to the meshes $m_{\sigma^{\prime}, j}$ of the set $B_{\sigma^{\prime}}$ is a set of intervals $I_{\sigma^{\prime}, j}$. We shall denote this set of intervals by $b_{\sigma^{\prime}}$. We shall show that if $P$ is any point of the mesh $m_{\sigma, i}$, then all of the corresponding points $y$ belong to $b_{\sigma^{\prime}}$. That is, if $y$ belongs to $C b_{\sigma^{\prime}}$, then $P_{y}$ belongs to $C m_{\sigma, i}$. Let $y$ be any point of $C b_{\sigma^{\prime}}$. Then $y$ belongs to some interval $I_{\sigma^{\prime}, j}$ and the corresponding point $P_{y}$ belongs to the corresponding set $\bar{m}_{\sigma^{\prime}, j}$. The set $\bar{m}_{\sigma^{\prime}, j}$ can be neither interior nor adjacent to $m_{\sigma, i}$. Hence $P_{y}$ belongs to $C m_{\sigma, i}$. Therefore if $s$ is sufficiently large, then $m\left(A_{s}^{\prime}\right)<\pi\left(m_{\sigma, i}\right)+\epsilon$. Since a similar inequality holds for every mesh of the lattice $L_{\sigma}$, it follows that if $s$ is sufficiently large, then $\pi\left(m_{\sigma, i}\right)-\epsilon<m\left(A_{s}^{\prime}\right)$. That is, $\lim _{s \rightarrow \infty} m\left(A_{s}^{\prime}\right)=\pi\left(m_{\sigma, i}\right)$. The extension to 
the case in which $A$ consists of a finite sum of meshes is immediate. Next let $A$ be an arbitrary set whose frontier points are of measure zero. Given any positive number $\epsilon$, there exist two elements $B_{1}$ and $B_{2}$ of $N$ such that $B_{1}<A<B_{2}$ and $\pi\left(B_{2}-B_{1}\right)<\epsilon$. Thus it is easily seen that $\lim _{s \rightarrow \infty} m\left(A_{s}^{\prime}\right)$ $=\pi(A)$.

The points $P_{s, j}$ are everywhere dense in the space $S$ and hence constitute a space $S^{\prime}$ defined in $\S 10$. There correspond a field $F^{\prime}$ and a distribution function $\pi^{\prime}(A)$. Let $A$ be any set of the field $F^{\prime}$ and let

$$
A_{s}^{\prime}=\sum_{P s, i \in A} m_{s, j}
$$

Then $\lim _{s \rightarrow \infty} m\left(A_{s}^{\prime}\right)=\pi^{\prime}(A)$. Let $A_{1}, A_{2}, \cdots, A_{\sigma}, E_{1}, E_{2}, \cdots, E_{h}$ be the sets involved in the operator $O_{t}$. There correspond sets $A_{s, 1}, A_{s, 2}, \cdots, A_{s, \sigma}$, $E_{s, 1}, E_{s, 2}, \cdots, E_{s, h}$ defined in accordance with equation (15). Let

$$
P[s, t, m(E)]=m\left(E_{s, 1}\right) \cdot m\left(E_{s, 2}\right) \cdots m\left(E_{s, h}\right) \cdot m^{-h}\left(A_{s, \sigma}\right) .
$$

Then $\lim _{s \rightarrow \infty} P[s, t, m(E)]=P[t, m(E)]$. If $y$ is any member of the set $G$ defined in $\$ 15$, then there is determined a sequence $K(y)$. There is also determined a corresponding sequence $K$ whose terms are elements of the space $S^{\prime}$. There exists an integer $s_{0}$ such that

$$
\left|p_{N}\left[O_{t}\left(K \subset x_{\mu, 1}\right)\right]-P[s, t, m(E)]\right|<2^{-s-1}
$$

whenever $s_{0} \leqq s, H(s-1, t) \leqq N \leqq J(s, y)$ and $\mu=\mu(s, y, t)$. Hence by reasoning similar to that of Theorem $2, \S 15$, it follows that $p\left[O_{t}(K)\right]=P[t, m(E)]$. That is, $K$ satisfies condition (C4) and hence is a collective with respect to the space $S^{\prime}$ and the field $F^{\prime}$. It is also a collective with respect to the space $S$ and the field $F$.

We shall now remove the restriction that $\pi(A)$ must be greater than zero whenever $m(A)$ is greater than zero. Let $\pi(A)$ be any absolutely additive, absolutely continuous distribution function such that there exists a set $B$ with the following properties.

1. The frontier points of $B$ are of measure zero, i.e., $B$ belongs to the field $F$.

2. $\pi(B)=1$.

3. If $A$ is any set of the field $F$, then $0<\pi(A \cdot B)$ whenever $0<m(A \cdot B)$. We shall define a new distribution function $\pi^{\prime}(A)$ by the equation

$$
\pi^{\prime}(A)=[\pi(A \cdot B)+m(A \cdot C B)] / 2 .
$$

Then there exist collectives having $\pi^{\prime}(A)$ as their distribution function. Let $K$ be such a collective and let us perform a contraction on $K$ with respect 
to the set $B$. The resulting sequence $K \subset \phi_{B}(K)$ is a collective having the distribution function

$$
\pi^{\prime}(A \cdot B) / \pi^{\prime}(B)=\pi(A) .
$$

Next if $S_{1}$ is the whole of the $\alpha$-dimensional euclidean space, then there exist collectives with respect to this space. To show this, it is only necessary to construct a transformation which is continuous and has a continuous inverse and which carries $S$ into $S_{1}{ }^{*}$ Such a transformation carries sets of measure zero into sets of measure zero.

Suppose we have a space $S$ which consists of a denumerable infinity of elements $m_{1}, m_{2}, m_{3}, \cdots$. Let the field $F$ contain all possible subsets of $S$. We shall define a corresponding set of intervals $I_{1}, I_{2}, I_{3}, \cdots$ as follows.

$$
\begin{array}{ll}
I_{j}: & \pi\left(m_{1}+m_{2}+\cdots+m_{j-1}\right) \leqq y<\pi\left(m_{1}+m_{2}+\cdots+m_{j}\right) \text { if } 1<j, \\
I_{1}: & 0 \leqq y<\pi\left(m_{1}\right) .
\end{array}
$$

Then $\sum_{j=1}^{\infty} I_{j}=I$ where $I$ is the interval $0 \leqq y<1$. If $A$ is any element of $F$, then the corresponding sum of intervals $I_{j}$ constitutes a set whose frontier points are of measure zero. Let $K^{\prime}=P^{(1)}, P^{(2)}, \cdots$ be any member of the set $C[D, m(E)]$ and let the corresponding label space be the interval $I$. We shall define a corresponding collective $K=e^{(1)}, e^{(2)}, e^{(3)}, \cdots$ having the label space $S$. If $P^{(n)}$ belongs to the interval $I_{j}$, then the corresponding term $e^{(n)}$ shall be the element $m_{j}$. Then $K$ is easily seen to be a collective. Any space consisting of a finite number of elements can be similarly treated.

We shall show the existence of collectives whose associated label space $S$ has infinitely many dimensions. We can express $S$ as the following infinite product.

$$
S=I_{1} \times I_{2} \times \cdots \times I_{\alpha} \times I_{\alpha+1} \times \cdots,
$$

where $I_{\alpha}$ is the interval $0 \leqq y_{\alpha}<1$. The field $F$ is defined as follows. A set $A$ belongs to $F$ if and only if there exists a number $\alpha$ such that $A=A_{\alpha} \times I_{\alpha+1}$ $\times I_{\alpha+2} \times \cdots$ and the frontier points of $A_{\alpha}$ are of measure zero.

For each interval $I_{\alpha}$ we shall construct a net $N_{\alpha}$ consisting of lattices $L_{s, \alpha}$. We shall also construct for the space $S$ a net $N$ consisting of lattices $L_{s}$. Let $L_{s}=L_{s, 1} \times L_{s, 2} \times \cdots \times L_{s, s}$. Then there are $2^{s^{2}}$ meshes $m_{s, 1}, m_{s, 2}, \cdots$, $m_{s, j}, \cdots, m_{s, 2^{s^{2}}}$ in $L_{s}$. We shall assume that the subscripts of the meshes are so chosen that

$$
m_{s-1, j+1}=\sum_{i=j \cdot 2^{2 s-1}+1}^{(j+1) 2^{2 s-1}} m_{s, i}
$$

* Copeland II. 
From this point on, the existence proof for collectives in this space is the same as that for collectives in the space of $\alpha$ dimensions.

Let $K=P^{(1)}, P^{(2)}, P^{(3)}, \cdots$ be a collective having the label space $S$. We shall express $K$ as a product collective. Let $k_{\alpha}$ be the sequence whose $n$th term $P_{\alpha}^{(n)}$ is the $\alpha$ th coordinate of the point $P^{(n)}$. Then $K=k_{1} \times k_{2} \times k_{3} \times \cdots$. Let $\alpha_{1}, \alpha_{2}, \alpha_{3}, \cdots$ be a sequence of positive integers and let $\beta_{n}=\alpha_{1}+\alpha_{2}+\cdots$ $+\alpha_{n-1}$. Let $S_{n}=S_{\beta_{n}+1} \times S_{\beta_{n}+2} \times \cdots \times S_{\beta_{n+1}}, K_{n}=k_{\beta_{n}+1} \times k_{\beta_{n}+2} \times \cdots \times k_{\beta_{n+1}}$. Then $K_{n}$ is a collective whose label space is $S_{n}$. Moreover $K_{n_{1}} \times K_{n_{2}} \times \cdots$ $\times K_{n_{\nu}}$ is a collective having the label space $S_{n_{1}} \times S_{n_{2}} \times \cdots \times S_{n_{\nu}}$. In the nomenclature of von Mises, collectives which can be combined in this manner to form new collectives are said to be "verbindbar." The spaces $S_{1}, S_{2}, \cdots$ and the collectives $K_{1}, K_{2}, \cdots$ can be transformed by one or another of the methods described earlier in this section. We shall denote the transformed spaces and collectives respectively by $S_{1}{ }^{\prime}, S_{2}{ }^{\prime}, S_{3}{ }^{\prime}, \cdots$ and $K_{1}{ }^{\prime}, K_{2}{ }^{\prime}, K_{3}{ }^{\prime}, \cdots$. The space $S$ and the field $F$ become $S^{\prime}$ and $F^{\prime}$. Products of the collectives $K_{1}^{\prime}, K_{2}^{\prime}, \cdots$ are collectives having the corresponding product label spaces.

17. Alternative systems. It is readily verified that $K_{i}^{\prime} \subset x$ and $K_{i}^{\prime}$ с $\phi_{A_{j}}\left(K_{j}^{\prime}\right)$ are collectives if $x$ is an element of $D$ and $A_{j}$ is a subset of $S_{j}^{\prime}$ and $I_{1}^{\prime} \times I_{2}^{\prime} \times \cdots \times I_{j-1}^{\prime} \times A_{j}^{\prime} \times I_{j+1}^{\prime} \times \cdots$ is an element of $F^{\prime}$. Operations of the form $K_{i}^{\prime} \subset \phi_{A_{j}}\left(K_{j}^{\prime}\right) \cdot \subset \cdot \phi_{A_{m}}\left(K_{m}^{\prime}\right)$ and $K_{i}^{\prime} \subset x_{1} \cdot \subset \cdot \phi_{A_{j}}\left(K_{j}^{\prime} \subset x_{2}\right)$ do not necessarily produce collectives. However it may be true that $K$ can be so chosen that all such operations and groups of these operations produce collectives. In fact in the system of Dörge, all of these operations are included.* Dörge considers a set of selections $S$ which is equivalent to the set $D$ given in this paper. He then constructs an extended set $\hat{S}$ which contains all the operators which can be formed by combining selections and contractions in the manner just described. Thus $\hat{S}$ includes all the operators of the form $O_{\sigma, t}$. In the present paper the operators $O_{t}$ play a rôle similar to those operators of the set $\hat{S}$ which are not of the form $O_{\sigma, t}$. In Dörge's system the spaces $S_{1}^{\prime}, S_{2}^{\prime}, \cdots$ consist of finite numbers of elements and the collectives $K_{1}^{\prime}, K_{2}^{\prime}, \cdots$ are independent. No general existence proof has been given for this system.

In the system of Reichenbach, $\dagger$ a fundamental element is an expression of the form $(i)\left(x_{i} \in 0 \ni_{p} y_{i} \in P\right)$. These elements admit of several interpretations. One of the most important interpretations is the following. Let

$$
\begin{aligned}
& x=x_{1}, x_{2}, x_{3}, \cdots, x_{i}, \cdots, \\
& y=y_{1}, y_{2}, y_{3}, \cdots, y_{i}, \cdots
\end{aligned}
$$

be two collectives with corresponding label spaces $S$ and $T$ and let $K=x \times y$ be

* Dörge I.

$\dagger$ Reichenbach I. 
a collective with the label space $S \times T$. Then the expression $(i)\left(x_{i} \in 0 \ni_{p} y_{i} \epsilon P\right)$ is equivalent to the equation $p\left\{\phi_{0 \times P}\left[K \subset \phi_{0 \times S}(K)\right]\right\}=p$. Another interpretation is obtained as follows. Let $y$ be a collective, $x$ be a selection of the set $D$, and 0 be the set consisting of the number 1 . Then $x=\phi_{0}(x)$. In this case the expression $(i)\left(x_{i} \epsilon 0 \ni_{p} y_{i} \epsilon P\right)$ means $p\left[\phi_{P}(K \subset x)\right]=p$.

If the collectives $K_{1}^{\prime}, K_{2}^{\prime}, \cdots$ are independent, then $K^{\prime}$ constitutes a Tornier matrix of a very general nature.* The $i$ th row of this matrix is the collective $K_{i}^{\prime}$. In the definition of such a matrix, conditions (C2) and (C3) are not required. Condition (C1) is specialized by setting $h=r_{1}=n=1$.

E. BOREL.

\section{REFERENCES}

I. Traité du Calcul des Probabilités. Edited by P. Dubreil. Paris, Gauthier-Villars, 1926.

A. H. Copeland.

I. The theory of probability from the point of view of admissible numbers, Annals of Mathematical Statistics, vol. 3 (1932), pp. 143-156.

II. Admissible numbers in the theory of geometrical probability, American Journal of MatheK. DÖRgE. matics, vol. 53 (1931), pp. 153-162.

I. $Z u$ der von $R$. von Mises gegebenen Begrïndung der Wahrscheinlichkeitsrechnung, MatheE. KAMKE. matische Zeitschrift, vol. 32 (1930), pp. 232-258.

I. Über neuere Begrïndungen der Wahrscheinlichkeitsrechnung, Jahresbericht der Deutschen Mathematiker Vereinigung, vol. 42 (1932), pp. 14-27.

II. Einfïhrung in die Wahrscheinlichkeitstheorie, Leipzig, Hirzel, 1932.

A. KolmogorofF.

I. Grundbegriffe der Wahrscheinlichkeitsrechnung, Ergebnisse der Mathematik, vol. 2, No. 3. C. DE la Vallee Poussin.

I. Sur l'intégral de Lebesgue, these Transactions, vol. 16 (1915), pp. 435-501.

C. I. Lewis AND C. H. LANGFoRd.

I. Symbolic Logic. New York, Century Publishing Co., 1934.

R. voN MrSEs.

I. Grundlagen der Wahrscheinlichkeitsrechnung, Mathematische Zeitschrift, vol. 5 (1919), pp. 52-99.

II. Wahrscheinlichkeit, Statistik und Wahrheit. Berlin, Springer, 1928.

III. Wahrscheinlichkeitsrechnung und ihrer Anwendung in der Statistik und theoretischen Physik. Leipzig and Vienna, Deuticke, 1931.

IV. Über Zahlenfolgen, die ein Kollektiv-ähnliches Verhalten zeigen, Mathematische Annalen, vol. 108 (1933), pp. 757-772.

F. REgan.

I. Applications of the theory of admissible numbers to time series with constant probability, these Transactions, vol. 36 (1934), pp. 511-529.

H. REICHENBACH.

I. Axiomatik der Wahrscheinlichkeitsrechnung, Mathematische Zeitschrift, vol. 34 (1931-32), pp. 568-619.

E. TORNIER.

I. Die Axiome der Wahrscheinlichkeitsrechnung, Crelle's Journal, vol. 163 (1930), pp. 45-64.

* Tornier I.

UNIVERSITY OF MichigAN, ANN ARbor, Mich. 